\section{$\underset{\substack{\text { hommes } \\ \text { \& migrations }}}{ }$}

\section{Hommes \& migrations}

Revue française de référence sur les dynamiques

migratoires

1317-1318 | 2017

L'Europe en mouvement

\title{
Donner la voix à qui ne l'a pas
}

Un spectacle de la compagnie La Maggese-Arsenal Fragile : Sur les traces de l'immigration italienne

\section{Michela Slapater}

\section{Q OpenEdition}

\section{Journals}

\section{Édition électronique}

URL : https://journals.openedition.org/hommesmigrations/3906

DOI : 10.4000/hommesmigrations.3906

ISSN : 2262-3353

Éditeur

Musée national de l'histoire de l'immigration

Édition imprimée

Date de publication : 1 avril 2017

Pagination : 132-138

ISBN : 978-2-919040-38-4

ISSN : $1142-852 X$

\section{Référence électronique}

Michela Slapater, «Donner la voix à qui ne l'a pas », Hommes \& migrations [En ligne], 1317-1318| 2017, mis en ligne le 01 avril 2017, consulté le 28 juin 2022. URL : http://journals.openedition.org/ hommesmigrations/3906 ; DOI : https://doi.org/10.4000/hommesmigrations.3906 
DONNER LA VOIX À QUI NE L'A PAS UN SPECTACLE DE LA COMPAGNIE LA MAGGESE-ARSENAL FRAGIL
DE L'IMMIGRATION ITALIENNE

Par MicheLA SLATAPER.

Les comédiens Margherita Trefoloni et Simone Olivi et la comédienne et metteure en scène Anna Andreotti expliquent leur démarche de collecte de la parole et des chants d'immigrés

à travers l'art, de la dimension universelle du phénomène migratoire. 
Hommes \& Migrations :

\section{Votre compagnie s'appelle " La Maggese » qui signifie la " jachère " en italien. Quel est le rapport entre les deux?}

Anna Andreotti : Au début, il s'agissait d'une boutade. Margherita qui est de Sienne et moi, qui suis née à Florence, nous nous sommes connues en France. En plus du théâtre, nous avions donc en commun une origine régionale mais aussi le mois de notre naissance, le mois de mai'. Rapidement cela a pris un sens beaucoup plus profond. Dans le travail que fait la compagnie, l'écriture a un rôle très important : il s'agit d'une écriture qui, depuis plusieurs années, est proche de la terre et du phénomène de " travail immobile " que réalise justement une jachère, une sorte de mise en pause et fructification dans le temps avant la collecte, c'est à dire la transmission orale des chants et des textes. Mais il y a aussi « i maggianti $i^{2}$ » qui sont une tradition théâtrale orale populaire, bien que de grands auteurs tels Luigi Pirandello ou Enrico Pea aient aussi écrit des « maggi ». Personnellement ce qui m'intéresse est la valeur du peuple et sa mémoire. Je viens d'un milieu plutôt « bourgeois »-du moins par ma mère - et aisé qui a toujours eu un grand respect pour le travail manuel et pour l'artisanat. 


\section{INITIATIVE}

Simone Olivi : Arsenal Fragile est une association née il y a une dizaine d 'années. Elle a fusionné avec La Maggese dont elle est aujourd'hui le support administratif. En plus de jouer, je m'occupe de la comptabilité, des budgets, des subventions et des contrats avec les mairies, les musées, les écoles... Quand on a fusionné on cherchait un nom pour une association théâtrale et on a trouvé « arsenal » parce que c'est le lieu où les choses se fabriquent, et " fragile " parce que le théâtre est très fragile. En plus c'est compréhensible en italien comme en français.

Margherita Trefoloni : La compagnie La Maggese existe depuis 1991. J'y suis arrivée en 2009. Au départ je faisais partie d'un groupe créé par Anna : Chants de rage et de révolte. II s'agissait de chants italiens de lutte qu'on chantait là où il y avait des revendications importantes, par exemple en faveur des sans-papiers. Ce qui m'intéressait personnellement c'était la fonction réactualisée du chant.

A. A. : Ensuite, le groupe ayant mûri au niveau des sonorités, j'ai proposé un projet militant ainsi est né : Sur les traces de l'immigration italienne.

H\&M : Lors de l'exposition Ciao Italia, nous verrons une " station ", angle de vue particulier sur cette immigration italienne qui a été un parcours de souffrance, de douleur et de déracinement avant de devenir un exemple d'intégration réussie, voire un motif de fierté pour les italiens de France, lorsque l'on évoque Lazare Ponticelli ou la famille Bugatti, pour ne citer qu'eux. Qu'est-ce une station ?

M. T. : Le spectacle se déroule par "stations" chantées et récitées, chacune autour d'une thématique. Ce sont les étapes du phénomène migratoire : le départ, la mémoire, l'oubli, le travail des femmes, la communauté (celle de
Anna Andreotti, 2016. @ FrançoIs RomAn / LAPETITECOURONNE.TV.

départ ou celle d'arrivée), et chaque "station " est constituée de témoignages recueillis auprès d'émigrés : chants et paroles...

\section{H\&M : Le choix du nom « station » a une valeur en lui même ...}

M. T. : Ça fait référence aux gares où arrivaient les émigrants, mais aussi au chemin de croix. Nous sommes arrivés à la onzième, celle de l'engagement politique.

S. O. : Dans notre parcours chanté pour l'exposition, nous avons choisi de présenter la neuvième "station ". C'est la plus aboutie car on nous a payé davantage pour la réaliser. Nous avons donc pu l'emmener en tournée un peu partout et nous l'avons travaillée plus longtemps. Elle contient aussi plus de chants de l'émigration que les autres et est présentée dans le langage des gens qui ont témoigné. 


\section{À droite : Dina N., née à Bettola (Plaisance) en 1923,} arrivée en France en 1946. @ VERONICA MECCHIA.

M. T. : C'est la "station de Dijon " et elle a une histoire particulière. Elle a été créée avec le chœur de Dijon qu'Anna a dirigé. Ensuite elle a voyagé et est revenue à Paris en 2010, puis à Nantes, et là elle voyage encore. Plusieurs chœurs sont impliqués dans cette station et se déplacent avec elle. Nous l'amènerons aussi à Rome pour travailler avec des anciens élèves de Giovanna Marini³. Ils ont aussi travaillé sur d'autres chants que nous avions collectés à Dijon. C'est un projet protéiforme qui grandit. Dans l'exposition, nous avons mis aussi un extrait de la "station » sur le travail des femmes : il s'agit du récit d'une "mondina ${ }^{4}$ » exploitée dans les rizières en Italie et au service de ses frères qui avaient un bistrot en France.

A. A. : C'était la double peine qui revient dans tous les témoignages des femmes émigrées ... 
H\&M : Comment procédez-vous pour recueillir ces témoignages ? Quelle est la méthode adoptée dans la collecte de la parole, des traces de l'émigration dans la mémoire des italiens ?

A. A. : J'ai appris en regardant Giovanna Marini et en travaillant avec elle à l'université Paris-VIII pendant cinq ans, après avoir fait des études d'anthropologie à Florence. Mais je suis comédienne et je travaille avec les émotions. C'est ce que j'utilise dans mon travail, rien d'autre. Je mobilise la même empathie quand j'aborde un personnage ou un texte pour le comprendre et pouvoir le jouer. Cela met les gens en confiance. Ce n'est peut-être pas une méthode très scientifique : j’ai bien sûr une idée de ce qui m'intéresse, mais je ne pars jamais d'un questionnaire écrit. La collecte a lieu au cours d'une discussion qui peut durer deux heures, parfois je peux y retourner deux, trois, quatre fois. Il y a une connivence avec celui qui témoigne du fait que moi aussimême si c'est pour une toute autre raison - je suis une déracinée et je travaille avec mon état émotionnel. Cela joue énormément pour les gens, tout comme le fait que j'ai l'âge de leurs enfants : tout ce qu'ils n'arrivent pas à leur dire, ils me le disent à moi. Pour déclencher la parole, je me mets beaucoup en jeu avec des anecdotes de ma vie. Je sais que ce n'est pas très orthodoxe, mais cela permet d'avoir de véritables échanges avec une sincérité que je n'aurais jamais avec un questionnaire.

\section{H\&M : La communauté dans laquelle vous avez fait vos enquêtes et vos retranscriptions de paroles est celle de Montreuil. Pourriez-vous nous expliquer en quoi consistent les " apéritifs italiens" de Montreuil ?}

A. A. : Tout a démarré avec un apéritif chez l'un des membres du chœur qui habite Montreuil. II y avait beaucoup d'Italiens de première, seconde et troisième génération. J'achetais un logement dans cette ville et je me suis rendu compte que le notaire avait un nom italien et qu'il y avait ici beaucoup de maisons "à l'italienne », c'est-à-dire faites avec les matériaux de là-bas, carrelées à l'italienne, avec le four à pain et pizza dans le jar- 
din et le balcon côté rue pour ce besoin absurde qu'ont les Italiens que tout le monde puisse les voir. À partir de là, j'ai décidé de commencer à enregistrer car je tenais un spectacle. Montreuil n'a été que le démarrage. Par la suite nous sommes allées là où il y avait une demande, par exemple à Charleville ou Dijon et Sète. En juin, je commencerai à « récolter » en Lorraine et à Metz, partout où il y a une communauté et où j’ai un contact.

\section{$H \& M$ : Il y a donc la phase de discussion et de récolte puis de retranscription ...}

A. A. : Oui, une chose est discuter. Après, au moment de retranscrire, je retranscris tout : hésitations, répétitions, erreurs... j'essaie de garder le plus possible la langue authentique. II y a ensuite le passage à la représentation. Au fur et à mesure, nous avons trouvé une méthode de travail. Quand j'ai commencé, la toute première fois, je me suis dit : "Je démarre, j'enregistre, après je vais voir ce que j'en ferai. » J'ai retranscrit et noté mes impressions dans un journal, puis je me suis demandée si cette parole était viable et j'ai vu que, pour la collecte, le moment de la représentation est fondamental. Après avoir témoigné et donné souvent l'image d'une immigration idéale qui "s'est très bien passée ", les gens qui viennent au spectacle (ils n'ont pas forcément l'habitude de se rendre au théâtre) à la fin de la représentation commencent à dire « moi aussi, moi aussi » et, à ce moment-là, on peut récolter la parole d'une histoire enfouie dans certaines familles, une histoire refoulée car très douloureuse. La représentation elle-même a donc une fonction dans la recherche.

\section{H\&M : Et c'est pour cela aussi que c'est très long, parce que souvent les choses ne sortent pas d'emblée, c'est peut-être au moment de partir, sur le pas de la porte, que les personnes se livrent ?}

A. A. : C'est pour cette raison que la collecte est difficile quand on est " extérieur ". II faut avoir un pied sur place, et au fur et à mesure on finit par comprendre comment il faut procéder.

M. T. : Quand celui qui a témoigné vient voir une « station » du spectacle, c'est comme une boucle qui se boucle. Il y a des étapes dans la démarche de collecte : la recherche, la prise de contact avec les gens, les témoignages, la retranscription, les chants enregistrés si les témoins chantent quelque chose, la retransmission du chant au chœur, notre présence avec notre propre vécu. Ces étapes se densifient progressivement jusqu'au spectacle et à la rencontre avec le public. L'ensemble prend alors encore plus d'épaisseur parce que le public participe, soit en nous racontant encore autre chose, soit en confirmant ce qu'il a vu sur scène. II s'agit d'un projet qui a plusieurs niveaux : artistique, humain, ethnomusicologique. 


\section{REPÉRAGE}

S. O. : Nous avons fait un spectacle pour l'association France-Frioul à la Maison de l'Italie. Ils nous avaient demandé de leur montrer notre travail et ont rempli la salle. C'était assez incroyable car, au moment où on ouvrait la bouche, on voyait les personnes sursauter et nous chantions avec un écho : il y avait un véritable dialogue entre nous trois et les 150 personnes dans la salle. Ce n'était même plus un spectacle...
A. A. : Exactement. Nos représentations ne sont jamais très "propres ", ni parfaites ou bien ficelées, parce que le chœur lui-même n'est pas parfait. Ce sont trente êtres humains : je ne veux pas des singes domptés. Ils participent parce que le projet les intéresse : ce que nous présentons sont à 80-90\% des chants collectés en France, des petits chants, mais c'est un patrimoine précieux et le chœur en est conscient. I 